\title{
Schnittstelle für Selbstbedienungsautomaten in RFID-Bibliotheken Vergleich der Schnittstellen SIP2 und NCIP
}

\author{
Barbara Michaelis
}

In RFID-Bibliotheken erfolgt die Kommunikation zwischen Selbstbedienungsautomat und Bibliothekssystem mittels standardisierter Protokolle. Zurzeit sind vor allem die Protokolle SIP2 und NCIP im Einsatz. Diese unterscheiden sich im strukturellen Aufbau und im Bereich der Definitionen grundlegend voneinander. Daraus ergeben sich wesentliche Einflüsse auf den Aufwand für die Installation und Wartung der Schnittstelle zwischen Selbstbedienungsautomaten und Bibliothekssystem.

\section{Kommunikation zwischen Selbstbedienungsautomat und LMS (Library Management System)}

Für den Einsatz von Selbstverbuchungsgeräten muss die Frage der Kommunikation zwischen diesem und dem Bibliothekssystem (LMS) gelöst werden. Dabei ist es unerheblich, mit welchen Lesevorgängen (Barcode, RFID oder Bilderkennung) gearbeitet wird. Die Geräte arbeiten heutzutage vorwiegend auf der Grundlage der RFID-Technologie. Selbstverbuchungsgeräte besitzen jeweils Programme für die Lösung ihrer Aufgaben. Ein integriertes Bibliothekssystem bedient eine Vielzahl von Arbeitsplätzen und Geräten. Die notwendigen Prozesse werden zentral auf Servern verwaltet. So ist es erforderlich, die Kommunikation von einem Selbstbedienungsgerät zur Serverapplikation des LMS zu gewährleisten. Das kann individuell gelöst werden. Der Nachteil individueller Lösungen besteht immer darin, dass diese für andere Konstellationen nicht anwendbar sind. So haben sich für die Kommunikation im bibliothekarischen Bereich seit langem verschiedene Protokolle entwickelt. Damit wird die Unabhängigkeit zwischen den Herstellern der Automaten und der Bibliothekssysteme weitgehend gewährleistet.

Damit Protokolle breite Anwendungsmöglichkeiten finden und auch über längere Zeiträume verwendbar sind, sollten sie folgende Eigenschaften besitzen:

- eindeutige Regeln und Definitionen

- Unterstützung sämtlicher Erfordernisse

- Stabilität

- Flexibilität und Weiterentwicklung

Mängel oder Lücken werden von den Anwendern selbst durch Modifizierung ausgeglichen. Damit entstehen Lösungen, die für andere Applikationen in dieser Form nicht mehr nutzbar sind. Beide Partner müssen sich immer wieder mit diesen Modifikationen auseinandersetzen. Damit erhöhen sich der Aufwand für die Hersteller sowie die Kosten für die Bibliotheken. 


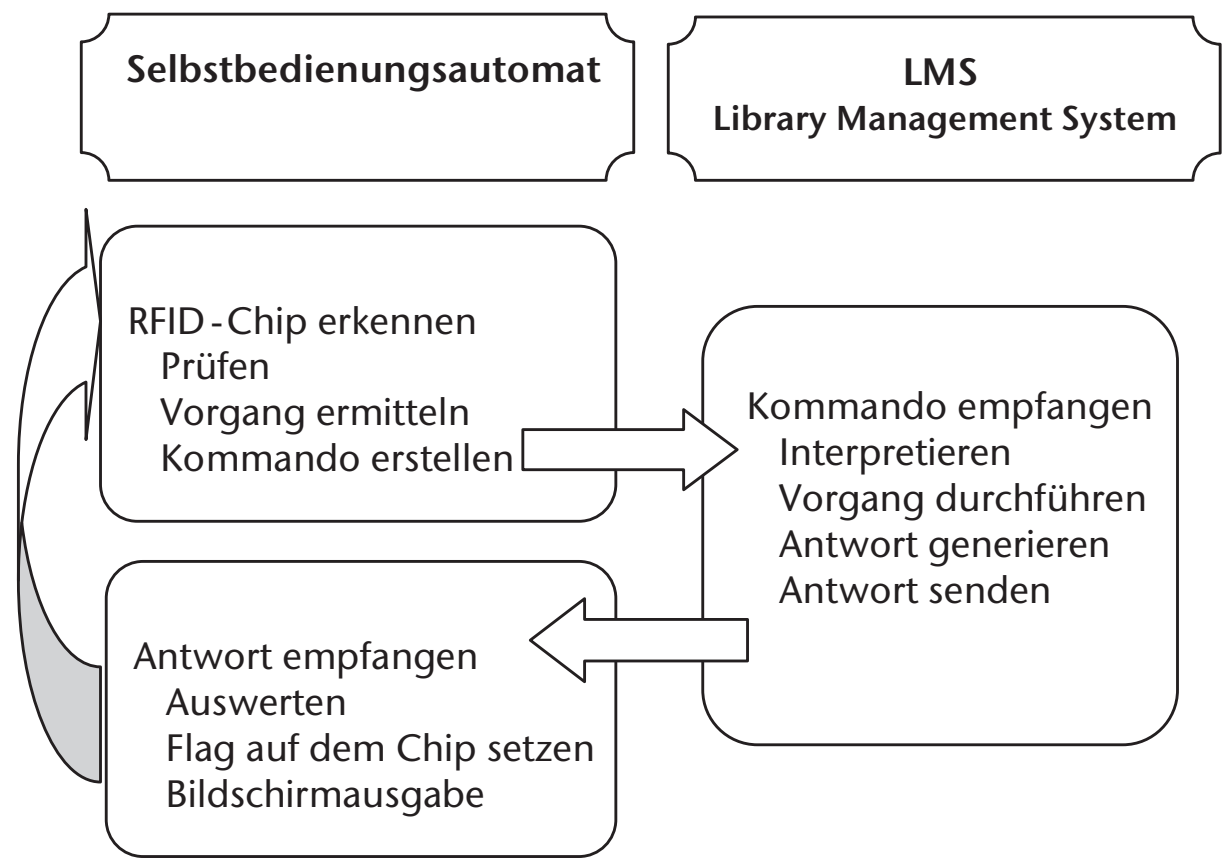

Abb. 1: Kommunikation: RFID-Selbstbedienungsautomat-Bibliothekssystem

\section{Welche Kommunikationsprotokolle existieren bereits?}

Ein Standard für die Selbstverbuchung wurde bereits 1997 von 3M entwickelt. Dieser Standard -SIP (Standard Interchange Protocol) -wurde dann von vielen Bibliotheken eingesetzt und ist als SIP2 (3M TM Standard Interchange Protocol Version 2.00) heute noch das am meisten verwendete Protokoll. Es wird fortlaufend weiter entwickelt.

Im Zuge der Entwicklung Web-basierter Bibliotheken und des ILL (Interlibrary Loan) entstand die Notwendigkeit des Austauschs von Daten von Bibliotheken, Benutzern und Exemplaren sowie auch der Ausführung von Operationen für Benutzer und Exemplare in anderen Bibliotheken. Auf der Grundlage des bereits vorhandenen SIP2-Protokolls wurde für die neuen Herausforderungen das NISO Circulation Interchange Protocol (NCIP-Z39.83) entwickelt. Die erste Version entstand im Jahre 2002. Dieses umfassende Protokoll beinhaltet auch die Funktionen, die für die Kommunikation zwischen Selbstverbuchern und LMS notwendig sind. Die aktuelle Version ist aus dem Jahr 2008.

Ein weiteres Protokoll ist SLNP (Simple Library Network Protocol) der Firma SISIS. Dieses wird vorwiegend in SISIS-SunRise-Anwendungspaketen für Fernleihserver in deutschen Bibliotheksverbünden eingesetzt und ist vom Funktionsumfang für die Selbstverbuchung nicht so gut geeignet.

Der Einsatz von API-Schnittstellen (application programming interface) ist natürlich auch denkbar. Hierbei sind dann jedoch umfassende individuelle Vereinbarungen notwendig. 


\section{Grundlagen der Protokolle SIP2 und NCIP}

Der Verbindungsaufbau für einen Prozess kann für beide Protokolle über TCP/IP (Transmission Control Protocol/ Internet Protocol) erfolgen. Die Verbindung über Web-Services (http bzw. https) ist nur für NCIP möglich. Eine verschlüsselte Verbindung über https ist somit nur bei Verwendung von NCIP möglich.

Die Verbindung selbst ist verbindungsorientiert (Connection oriented). Damit entstehen zeitweise Interaktionen zwischen den Partnern in Realzeit. Das Selbstbedienungsgerät setzt einen Request (Anfrage) ab und das LMS beantwortet den Request sofort.

Die Requests werden prozessunabhängig ausgeführt, d. h. jede Interaktion ist unabhängig von vorigen Aktionen. Damit kann eine Verbindung ohne nachfolgende Probleme nach jeder Interaktion enden. Für eine weitere Interaktion wird dann eine neue Verbindung automatisch aufgebaut.

\section{Protokollsprachen}

\section{SIP2}

Hier werden Request und Response (Antwort) in strukturierter Form im sogenannten Package-Format übergeben.

Request und Response beginnen mit einem eindeutigen Command-Identifier. Danach werden die Inhalte der Felder mit definierten, festen Längen in vorgegebener Reihenfolge übergeben. Zum Schluss werden Felder mit variabler Länge und definierten Feldindikatoren übergeben.

Auf Grund der linearen Struktur lassen sich multiple Angaben nicht abbilden.

Definierte Vorgaben für die Belegung sind nur an wenigen Stellen vorhanden. Damit ergeben sich vielfältige Möglichkeiten der Belegung. Kommandos müssen deshalb herstellerabhängig ausgewertet und beantwortet werden.

\section{NCIP}

Request und Response werden im XML-Format (Extensible Markup Language) übergeben. Damit existieren jeweils genaue Beschreibungsmöglichkeiten in Form einer DTD (Document Type Definition). Request und Response können mit sogenannten Parsern jeweils überprüft werden. Für den strukturellen Aufbau existiert ein eindeutiges XMLRegelwerk.

Hierarchische Strukturen, die für multiple Angaben so wichtig sind, lassen sich hierbei genau abbilden. Hat ein Benutzer zum Beispiel verschiedene Sperren, die eine Ausleihe nicht zulassen, so können hier alle Gründe detailliert bekanntgegeben werden. Auch unterschiedliche Gebühren können nur unter Verwendung hierarchischer Strukturen genau abgebildet werden. 
NCIP verwendet spezifizierte Datentypen (Enumerated Data Types). Erweiterungen müssen in Profilen bekannt gegeben werden. Muss das LMS zum Beispiel eine Aktion (Ausleihe, Rückgabe oder Verlängerung) ablehnen, so gibt es pro Request eine Menge von zulässigen Fehlermeldungen. Für diese sind keinerlei individuelle Absprachen und Umsetzungen notwendig. Die Aufgabe des Selbstbedienungsautomaten besteht dann darin, diese Meldungen in die gewünschte Sprache bibliotheksspezifisch umzusetzen.

\section{Signifikante Unterschiede}

\section{Ausgabe komplexer Informationen}

Mit SIP2 können komplexe Angaben in individueller Form übergeben werden. Die Angaben für die Sortierung, die Bildschirmausgabe oder die Ausgabe auf einem Quittungsdrucker können beliebig mit »sort bin«, »screen message«bzw. »print line « übergeben werden. Das ist für die Realisierung in einer Bibliothek sehr bequem, jedoch müssen diese für jede Bibliothek individuell angepasst werden. Mit NCIP müssen diese Angaben detailliert und typgerecht angegeben werden.

So ergibt sich aus dem NCIP-Protokoll eine strikte Trennung der Aufgaben zwischen Automat und LMS. Das LMS übergibt strukturierte, definierte Daten und der Automat bildet daraus die entsprechenden Ausgaben für Bildschirm und Drucker. Diese Trennschärfe ist bei Verwendung von SIP2 so nicht erkennbar.

Für die Definition einer Sortieranlage ist es zudem günstiger, aus mehreren, strukturierten NCIP-Angaben eines Exemplars die richtige Sortierbox jeweils im entsprechenden Rückgabeautomaten zu ermitteln. Da in einem Bibliothekssystem mit unterschiedlichen Standorten auch unterschiedliche Rückgabeautomaten mit abweichenden Sortierboxen vorhanden sein können und sich diese Sortiervorgaben auch ändern können, sollten diese Sortiereinstellungen am Rückgabeautomaten vorgenommen werden können. Günstig sind dabei Einstellungen, die keine Programmänderung erfordern und die die jeweilige Bibliothek selbst vornehmen kann.

\section{Authentifizierung}

Mit NCIP kann im Request genau angegeben werden, wie die Authentifizierung erfolgen soll. Genaue Angaben, ob die Anmeldung mit oder ohne Passwort und mit Ausweisnummer oder einer anderen Nummer erfolgen soll, sind hier möglich. Diese Einstellungen fehlen bei SIP2. Hier können nur "patron identifier « und »password identifier « angegeben werden. Die Definition, was der "patron identifier« ist, unterliegt der genaueren Absprache zwischen den Herstellern der Automaten und des LMS. 


\section{Kennzeichnung eines Exemplars}

Auch hier kann im NCIP-Request genau angeben werden, mit welchen Angaben das Exemplar ermittelt werden soll. Das kann die eindeutige Mediennummer aber auch die UID des RFID-Chips sein. Im SIP2-Request ist hierzu keine spezifizierte Angabe möglich.

\section{Anzeige eines Exemplars}

Strukturierte Titelinformationen wie Titel, Autoren, Verlag, Erscheinungsjahr usw. lassen sich nur mit NCIP erzeugen. In SIP2 gibt es mit »title identifier« keine definierte Ausgabe.

\section{Anzeige Benutzerkonto}

Mit NCIP können Ausleihen oder Bestellungen einzeln mit vielen Angaben strukturiert und mit fest definierten Werten ausgegeben werden. Bei SIP2 ist die Ausgabe unstrukturiert und damit individuell gestaltet.

Strukturierte Angaben von Gebühren, Sperren und Adressangaben sind nur mit NCIP möglich.

\section{Alle Exemplare verlängern}

Bei NCIP gibt es keine Möglichkeit, alle ausgeliehenen Exemplare eines Benutzers mit einem Request zu verlängern. Jedes Exemplar muss einzeln mit einem Request verlängert werden. In SIP2 steht für die Gesamtverlängerung der Request »renew all« zur Verfügung.

\section{Vorgang Ausleihe oder Rückgabe widerrufen}

Wenn ein Ausleihvorgang wieder rückgängig gemacht werden soll, weil der Benutzer z. B. das Exemplar zu schnell vom RFID-Reader entfernt hat und der Schreibvorgang für das AFI-Flag für die Sicherung der Medien nicht erfolgen konnte, kann das mit dem NCIP-Request »UndoCheckout« erfolgen. Für die Rückgabe steht ein solcher Request nicht zur Verfügung. Man geht davon aus, dass im Rückgabeautomaten ein Exemplar kaum vom Benutzer wieder »herausgeholt« werden kann. Mit SIP2 gibt es dagegen »cancel« sowohl für die Ausleihe als auch die Rückgabe.

\section{Fazit}

Sowohl SIP2 als auch NCIP sind für die Gestaltung der Schnittstelle zwischen Selbstverbuchungsautomat und LMS geeignet. Mit SIP2 lassen sich jedoch nur Lösungen erzielen, die mit individuellen Absprachen zwischen den Herstellern der Software für die Automaten und des LMS einhergehen. Das kann für beide Partner mit einem hohen Zeitaufwand verbunden sein. Dabei kann sich der Kostenaufwand für die Hersteller und damit auch für die Bibliothek erheblich erhöhen. Nach dem nun für die RFID-Tags selbst ein Standard (dänisches Datenmodell für Bibliotheken) entwickelt wurde, wäre es wünschenswert, die Standardisierung der Schnittstelle zwischen Automaten und LMS 
voranzutreiben. Unsere Erfahrungen als Entwickler von Bibliothekssoftware zeigen, dass die Verwendung des NCIP-Protokolls bei Implementationen für verschiedenste Bibliotheken die Arbeit wesentlich erleichtert hat.

Auch über die reine Selbstverbuchung hinaus ist dieses Protokoll bestens geeignet, selbst komplizierte Bezahlfunktionen am Kassenautomaten zu bedienen. Eine weitere Möglichkeit besteht in der Anfrage fremder Bibliotheken nach bestellbaren, verfügbaren Exemplaren zu einem gewünschten Titel. Dieses kann in Verbundsystemen als Vorstufe für die Fernleihe genutzt werden.

Für ein integriertes Bibliothekssystem betrachten wir es als selbstverständlich, die RFIDTechnologie auch in die Arbeitsabläufe im Bibliotheksystem selbst zu integrieren. Das betrifft die Vorgänge Konvertieren, Löschen sowie alle weiteren Bearbeitungsfunktionen von Exemplaren.

\section{Literatur und Internetquellen}

[1] 3M ${ }^{\mathrm{TM}}$ Standard Interchange Protocol, http://solutions.3m.com/wps/portal/3M/en_US/library/ home/resources/protocols/

[2] ANSI/NISO Z39.83-1 und ANSI/NISO Z39.83-2, http://www.niso.org./kst/reports/standards

[3] Barbara Michaelis (2009): Vergleich der Schnittstellen SPI2 und NCIP (PDF), http://www.th-wildau.de/hochschule/einrichtungen/bibliothek/die-bibliothek-vor-ort/ veranstaltungen-events/2-wildauer-symposium-rfid-und-medien.html

Die zitierten Internetquellen wurden zuletzt am 14.05.2010 aufgerufen. 